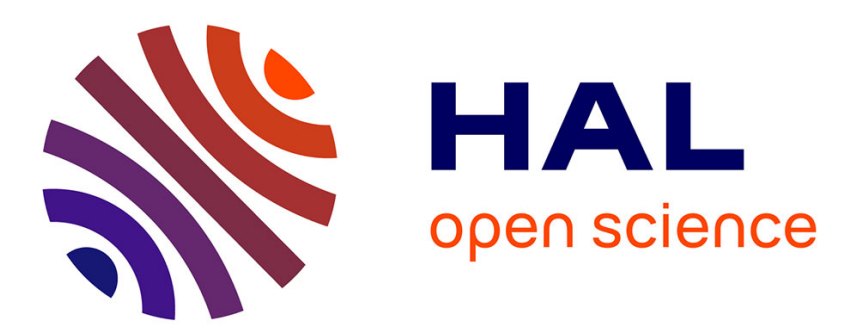

\title{
Algorithm to compute nonlinear partial observer normal form with multiple outputs
}

Wided Saadi, Driss Boutat, Gang Zheng, Lassaad Sbita, Lei Yu

\section{To cite this version:}

Wided Saadi, Driss Boutat, Gang Zheng, Lassaad Sbita, Lei Yu. Algorithm to compute nonlinear partial observer normal form with multiple outputs. IEEE Transactions on Automatic Control, 2020, 65 (6), pp.2700 - 2707. 10.1109/TAC.2019.2946528 . hal-02406825

\section{HAL Id: hal-02406825 \\ https://hal.inria.fr/hal-02406825}

Submitted on 12 Dec 2019

HAL is a multi-disciplinary open access archive for the deposit and dissemination of scientific research documents, whether they are published or not. The documents may come from teaching and research institutions in France or abroad, or from public or private research centers.
L'archive ouverte pluridisciplinaire HAL, est destinée au dépôt et à la diffusion de documents scientifiques de niveau recherche, publiés ou non, émanant des établissements d'enseignement et de recherche français ou étrangers, des laboratoires publics ou privés. 


\title{
Algorithm to compute nonlinear partial observer normal form with multiple outputs
}

\author{
Wided Saadi, Driss Boutat, Gang Zheng, Lassaad Sbita and Lei Yu
}

\begin{abstract}
It is well-known that observer design is a powerful tool to estimate the states of a dynamical system. Given a multi-output nonlinear dynamical system whose states are partially observable, this paper investigates the problem of observer design to estimate those observable states. It considers firstly a nonlinear system without inputs, and provides a set of geometric conditions, guaranteeing the existence of a change of coordinates which splits the studied nonlinear dynamical system into two subsystems, where one of them is of the well-known nonlinear observer normal form, for which a Luenberger-like observer can be designed. An extension to nonlinear systems with inputs has then been deduced.
\end{abstract}

Index Terms-Partially observable, observer design, differential geometric method

\section{INTRODUCTION}

A considerable amount of research has been done on observer error linearization problem for a nonlinear dynamical system (see e.g. [1], [2], [3] and the references herein). One of well-known approaches is to seek a change of coordinates that transforms the studied system into the so-called nonlinear observer normal form. This latter form enables us to easily design an observer which generates a linear error dynamics. This concept was firstly addressed by [2] for single-output dynamical systems described as

$$
\begin{aligned}
& \dot{x}=f(x) \\
& y=h(x)
\end{aligned}
$$

with $y \in \mathbb{R}$. For such a system, they established necessary and sufficient conditions that guarantee the existence of a change of coordinates $z=\phi(x)$ which transforms (1-2) into the following famous nonlinear observer output injection normal form

$$
\begin{aligned}
\dot{z} & =A z+\beta(y) \\
y & =C z
\end{aligned}
$$

where $A$ and $C$ are of the well-known Brunovsky observable canonical form. It is important to note that the proposed change of coordinates $z=\phi(x)$ linearizes the output of (2) as well. In order to enlarge the class of dynamical systems that can be transformed into a nonlinear observer normal form, one can also apply a diffeomorphism on the output of the form $\bar{y}=\varphi(y)$, which will lead to a similar observer normal form as follows: $\dot{z}=A z+\beta(\bar{y}), \bar{y}=C z$, where $\bar{y}=\varphi(y)$. We can see that, even if $\varphi$ is a nonlinear function of the output $y$, it does not cause problems when designing a Luenberger-like observer. This idea was firstly proposed in [4] to relax the linearization constraint on the output for the multi-output nonlinear system. A few years later, [3] provided further information and clarifications on the work of [4], [5] while limiting only to the case $\varphi=I$.

Manuscript received February $\mathrm{xx}, 20 \mathrm{xx}$; revised February $\mathrm{xx}, 20 \mathrm{xx}$ and September xx, 20xx; accepted September xx, 2019. Date of publication January xx, 2020; date of current version July xx, 20xx. Recommended by Associate Editor Xxxxxx xxxx. (Corresponding Author: Gang Zheng)

G. Zheng is with School of Mathematics and Big Data, Foshan University, Foshan 528000 China, and Inria Lille, 40 Avenue Halley, 59650 Villeneuve d'Ascq, France (gang.zheng@ @inria.fr).

W. Saadi and L. Sbita are with ENIG, Tunisia, D. Boutat is with INSA Centre Val de Loire, Université d'Orléans, PRISME EA 4229, Bourges Cedex 18022 France, and L. Yu is with Wuhan University, China.
One can also find the works of [6], [7] for a geometric characterization and the works of [8], [9] for an algebraic characterization. Other related works on this topic dealt with the problem of the linearization of the error dynamics by the so-called generalized inputoutput injection or direct transformation (see eg. [10], [11]). Besides, there exist as well some works treating the case where the above matrices $A$ and $C$ in (3-4) are of the Brunovsky form depending on the output (see eg. [12], [13], [14], [15]). A generalization of the work [3] was done in [16] for a triangular form of the output diffeomorphism $\varphi$. Within the framework of full-order nonlinear observer normal form, we can also refer to the works for the extended nonlinear observer normal form ([17], [18], [19], [20], [21], [22], [23]).

Up to now, most of research on this topic assumed that the whole state of the studied system is observable, for the purpose of transforming the whole system into the so-called observability normal form. Few works have been done for the partial observability case, which makes sense in many practical problems when only a part of states are observable for the controller design. To our knowledge, one of the first works to consider this problem is [24], where the authors provided necessary and sufficient geometric conditions which guarantee the existence of a change of coordinates transforming a singleoutput nonlinear dynamical system into a special partial observer normal form. These conditions are improved in [25]. Unfortunately, the proposed form turns out to be very restrictive since it is a direct extension of the result proposed by [2]. To overcome this weakness, [20] introduced a new partial observer normal form, which however treated only single-output nonlinear dynamical system.

We would like to emphasize that the objective of this type of transformation method is to facilitate the design of observer, i.e., re-using the well developed observers in the literature for the transformed normal form. If the whole state of the studied system is observable, one can design either full-order observer or reduced-order observer [26]. The concept of reduced-order observer is to decompose the studied dynamical system into two parts: the part of measured states (i.e., outputs) and those to be estimated, and the reduced-order observer aims at estimating only the second part of the decomposed system. This concept has been applied in [27], [28] and references therein for nonlinear systems.

Although reduced-order observers estimate only partial observable states for a fully observable system, the result presented in this paper is completely different, since it treats partially observable dynamical system. For linear system, via a linear transformation related to observability indices, Kalman decomposition [29] enables us to decompose the studied system into observable and non-observable parts. For nonlinear system, a similar result exists as well: by introducing a diffeomorphism defined by observability indices, the nonlinear system can be decomposed into observable and non-observable parts [5]. It should be noted that the observable part of the transformed system, by applying those two methods, is not of output injection form (3-4), but of classical Brunovksy form with nonlinearity in the last row of the observable part. Such a classical form cannot admit a simple Luenberger-like observer. Unlike those methods, this paper proposes geometric conditions that guarantee the existence of a change of coordinates that decomposes a partially observable multi- 
output nonlinear dynamical system into two subsystems, where the first dynamics is of output injection normal form involving observable state, whereas the second subsystem contains all observable and unobservable states. The advantage of such a decomposition is that we can design a simple Luenberger-like observer for the studied system.

In fact, this paper attempts to complete our previous paper [30] for multi-output nonlinear dynamical system by clarifying the important dependence property and giving complete proofs of results. Particularly, this paper gives corrections of Theorem 1 stated in [30] where the conditions were wrong, since it was an intuitive translation of the result obtained in [20] for the single-output case. For this, Proposition 1 in this paper is introduced. Moreover, in this work we give two examples to explain more clearly our results and to show that the multi-output case is quite different with respect to the single-output case studied in [20]. Also, an extension to nonlinear systems with inputs has also been presented in this paper.

\section{NOTATIONS AND PROBLEM STATEMENT}

This section provides notations and assumptions which are needed in this work. Let us consider a multi-output nonlinear dynamical system described as follows:

$$
\begin{aligned}
& \dot{x}=f(x) \\
& y=h(x)
\end{aligned}
$$

where $x \in \mathscr{X} \subset \mathbb{R}^{n}, f: \mathscr{X} \subset \mathbb{R}^{n} \rightarrow \mathbb{R}^{n}$ and $h: \mathscr{X} \subset \mathbb{R}^{n} \rightarrow \mathbb{R}^{m}$. Without loss of generality, it is assumed that $0 \in \mathscr{X}$ is an open subset of $\mathbb{R}^{n}, f(0)=0$ and $h(0)=0$. Furthermore, we assume that the components of $h: y_{i}=h_{i}$ for $1 \leq i \leq m$ are independent. Thus the co-distribution $D_{0}=\operatorname{span}\left\{d h_{i}\right.$, for $\left.1 \leq i \leq m\right\}$ spanned by the differential of the outputs satisfies $\operatorname{rank} D_{0}=m$. Set $m_{0}=m$ and $\theta_{i, 1}=$ $d h_{i}$ for $1 \leq i \leq m$. Then, for $k \geq 1$ we define the following family of co-distribution $D_{k}=\operatorname{span}\left\{\theta_{i, j}\right.$, for $\left.1 \leq i \leq m, 1 \leq j \leq k+1\right\}$ where $\theta_{i, j}=d L_{f}^{j-1} h_{i}$ denotes the $(j-1)^{\text {th }}$ Lie derivative of the $i^{\text {th }}$ output in $f$ direction, with $d L_{f}^{0} h_{i}=d h_{i}=\theta_{i, 1}$. Now, for $j \geq 1$, we have $D_{j-1} \subseteq$ $D_{j}$, and let us note $m_{j}=\operatorname{rank} D_{j}-\operatorname{rank} D_{j-1}$. Then, we can define the observability indices of the pair $(f, h)$ as the $m$-tuple $\left(r_{1}, \cdots, r_{m}\right)$ of integers where $r_{i}=\max \left\{j: m_{j} \geq i\right\}$ (see [31]). With a possible reordering of the outputs indices, it can be assumed that $r_{1} \geq r_{2} \geq$ $\cdots \geq r_{m}$. In this paper, the following assumption is imposed ([31]):

Assumption 1: The observability indices $\left\{r_{i}\right\}_{1 \leq i \leq m}$ are assumed to be constant on the open set $\mathscr{X}$ and satisfy the partial observability rank condition, i.e., $r=\sum_{i=1}^{m} r_{i} \leq n$. If $r=n$, then we have the full observability rank condition, i.e. the whole states are observable. Otherwise, there is an unobservable sub-manifold of the state space $\mathscr{X}$. In this case we say that the dynamical system is partially observable.

For the partial observability case, this paper treats the following problem.

Problem statement: Under Assumption 1, deduce necessary and sufficient geometric conditions that guarantee the existence of a change of coordinates $\phi(x)=\left(z^{T}, \eta^{T}\right)^{T}$ where $z=\left(z_{1}, \cdots, z_{m}\right)^{T}$ with $z_{i}=\left(z_{i, 1}, \cdots, z_{i, r_{i}}\right)^{T}$ for $1 \leq i \leq m$ and $\eta=\left(\eta_{1}, \cdots, \eta_{n-r}\right)^{T}$ such that the nonlinear dynamical system (5-6) can be transformed into the following normal form with two decoupled subsystems:

$$
\begin{aligned}
\dot{z}_{i} & =A_{i} z_{i}+\beta_{i}(\bar{y}) \\
\dot{\eta} & =\xi(z, \eta) \\
\bar{y}_{i} & =C_{i} z_{i}
\end{aligned}
$$

where $C_{i} z_{i}=z_{i, r_{i}}=y_{i}+\varphi\left(y_{1}, \cdots, y_{i-1}\right), A_{i}$ and $C_{i}$ are respectively the $\left(r_{i} \times r_{i}\right)$ and $\left(1 \times r_{i}\right)$ well-known Brunovsky forms:

$$
A_{i}=\left[\begin{array}{cccc}
0 & \cdots & 0 & 0 \\
1 & \cdots & 0 & 0 \\
\vdots & \ddots & \vdots & \vdots \\
0 & \cdots & 1 & 0
\end{array}\right], C_{i}=\left(\begin{array}{llll}
0 & \cdots & 0 & 1
\end{array}\right) .
$$

The reason to propose such a normal form (7-9) is that we can easily design a Luenberger-like observer for the subsystem (7) as follows:

$$
\dot{\hat{z}}_{i}=A_{i} \hat{z}_{i}+\beta_{i}(\bar{y})+K_{i}\left(y_{i}-C_{i} \hat{z}_{i}\right)
$$

where $K_{i}$ are the gains to be chosen such that $\left(A_{i}-K_{i} C_{i}\right)$ is Hurwitz. Accordingly, the observation error $e_{i}=z_{i}-\hat{z}_{i}$ satisfies the following linear dynamics: $\dot{e}_{i}=\left(A_{i}-K_{i} C_{i}\right) e_{i}$, for $1 \leq i \leq m$. It is clear that the observable states $z_{i}$ of (7) can be exponentially estimated via the above Luenberger-like observer.

In order to deduce the required change of coordinates $\phi(x)$, the following notations are introduced. For $\theta_{i, j}=d L_{f}^{j-1} h_{i}$, define the following co-distribution

$$
\Delta=\operatorname{span}\left\{\theta_{i, j}, \text { for } 1 \leq i \leq m \text { and } 1 \leq j \leq r_{i}\right\}
$$

Under Assumption 1, the above co-distribution is of dimension $r=$ $\operatorname{rank} \Delta=\sum_{i=1}^{m} r_{i} \leq n$, implying that $d L_{f}^{k} h_{j} \in \Delta$ for any $k$.

If $\operatorname{dim} \Delta=n$, we have the fully observable case which has been treated in [4] and [3]. This paper is interested in the partially observable case, i.e., $\operatorname{dim} \Delta<n$, then the annihilator (or kernel) of $\Delta$, defined as follows:

$$
\Delta^{\top}=\{X: v(X)=0, \text { for any } v \in \Delta\}
$$

is a distribution of dimension $n-r$.

Before ending this section, we are going to introduce an important family of vector fields $\left(\bar{\tau}_{i, j}\right)$ for $1 \leq i \leq m$ and $1 \leq j \leq r_{i}$, with $\sum_{i=1}^{m} r_{i}=r=\operatorname{dim} \Delta$, which will be used to construct the change of coordinates. The first $m$ vector fields $\bar{\tau}_{i, 1}$ for $1 \leq i \leq m$ are chosen as one solution of the following algebraic equations:

$$
\begin{aligned}
\theta_{i, r_{i}}\left(\bar{\tau}_{i, 1}\right) & =1, \text { for } 1 \leq i \leq m \\
\theta_{i, k}\left(\bar{\tau}_{i, 1}\right) & =0, \text { for } 1 \leq k \leq r_{i}-1 \\
\theta_{j, k}\left(\bar{\tau}_{i, 1}\right) & =0, \text { for } j<i \text { and } 1 \leq k \leq r_{i} \\
\theta_{j, k}\left(\bar{\tau}_{i, 1}\right) & =0, \text { for } j>i \text { and } 1 \leq k \leq r_{j}
\end{aligned}
$$

It is worth noting that the solution of (13-16) is not unique for the following two key reasons:

1) The non uniqueness might exist even for the fully observable case, i.e. $r=\sum_{i=1}^{m} r_{i}=n$. It can be explained by the number of the algebraic equations for which the vector field $\bar{\tau}_{i, 1}$ should satisfy. Indeed, the first vector field $\bar{\tau}_{1,1}$ may satisfy $r$ algebraic equations (if $r=n$, then it is unique), while the other $\bar{\tau}_{i, 1}$ need only fulfill $i r_{i}+\sum_{k=i+1}^{m} r_{k}=r-\sum_{j<i}^{m}\left(r_{j}-r_{i}\right)+\sum_{k=i+1}^{m} r_{k}$ algebraic equations;

2) For the case where $r<n$, one can notice that even $\bar{\tau}_{1,1}$ is not unique. For a given solution $\bar{\tau}_{i, 1}$ of the above algebraic equation, it can be seen that $\bar{\tau}_{i, 1}+H$ is also a solution of (13-16) for any $H \in \Delta^{\top}$. However, we observe that those solutions are defined modulo the distribution $\Delta^{\top}$.

For each family of vector fields $\bar{\tau}_{i, 1}$ for $1 \leq i \leq m$ fulfilling (13-16), we can calculate the rest of vector fields by induction as follows:

$$
\bar{\tau}_{i, k}=-\operatorname{ad}_{f} \bar{\tau}_{i, k-1} \text {, for } 1 \leq i \leq m \text { and } 2 \leq k \leq r_{i}
$$

where $\operatorname{ad}_{f} \bar{\tau}_{i, k-1}=\left[f, \bar{\tau}_{i, k-1}\right]$ denotes the Lie bracket of $f$ with $\bar{\tau}_{i, k-1}$. 
By using those vector fields and the associated 1-forms, the objective is to deduce a change of coordinates $\phi(x)$, such that its differential (noted as $\phi_{*}$ ) yields $\phi_{*}(f)=\left[\begin{array}{c}A_{i} z_{i}+\beta_{i}(\bar{y}) \\ \xi(z, \eta)\end{array}\right]$. The details will be presented in Section IV, and in Section III we would like firstly clarify the key difference when treating nonlinear systems with single output and multiple outputs.

\section{PRELIMINARY RESUlt}

Unlike single-output dynamical systems, the normal form for nonlinear systems with multiple outputs may have a link between the outputs and their high-order derivatives. This phenomenon becomes an obstruction to design a Luenberger-like observer for such a normal form. The purpose of this section is to clarify the conditions that enable us to avoid this situation. To achieve this goal, we will firstly recall the classical results for nonlinear multi-output observer normal forms investigated in [4], [3] and [16], and then discuss the above issue of dependence between an output and the derivative of others. To do so, consider the following set:

$$
£_{i}=\left\{d L_{f}^{k-1} h_{j}, \text { for } 1 \leq j \leq m \text { and } 1 \leq k \leq r_{i}\right\} \backslash\left\{d L_{f}^{r_{i}-1} h_{i}\right\}
$$

for $1 \leq i \leq m$, where the symbol \means exclusion. Note as well $£=\left\{\bar{\theta}_{i, j}\right.$, for $1 \leq i \leq m$ and $\left.1 \leq j \leq r_{i}\right\}$, thus $\Delta=\operatorname{span}\{£\}$ where $\Delta$ is defined in (11).

Assume that the studied dynamical system (5-6) is fully observable, i.e., $\operatorname{dim} \Delta=r=n$. Then the following result was stated in [4], [3].

Theorem 1: There exists a local diffeomorphism $z=\phi(x)$ which transforms the dynamical system (5-6) into the form (7-9) without the dynamics (8), if and only if

1) there exists a frame $\bar{\tau}_{j, s}$ given by equations (13-17) such that:

$$
\left[\bar{\tau}_{i, k}, \bar{\tau}_{j, s}\right]=0
$$

2)

for all $1 \leq i, j \leq m, 1 \leq k \leq r_{i}$ and $1 \leq s \leq r_{j}$;

$$
\operatorname{span}\left\{£_{i}\right\}=\operatorname{span}\left\{£_{i} \cap £\right\}
$$

where $f_{i}$ for $1 \leq i \leq m$ are defined in (18).

The condition (20) in Theorem 1 ensures that the outputs do not influence each other by their derivatives. To clarify this fact, assume that there exists $1 \leq i \leq m$ such that $\operatorname{span}\left\{£_{i}\right\} \neq \operatorname{span}\left\{£_{i} \cap £\right\}$, then there exists $j>i$ with $r_{i}>r_{j}$ and $1 \leq k \leq r_{i}-r_{j}$ such that

$$
d L_{f}^{r_{j}-1+k} h_{j} \notin \operatorname{span}\left\{£_{i} \cap £\right\}
$$

Since the studied system is assumed to be fully observable, we have

$$
d L_{f}^{r_{j}-1+k} h_{j}=\sum_{s=1}^{i-1}\left(\sum_{t=1, r_{s}>r_{i}}^{r_{s}-r_{i}} \lambda_{s, r_{i}+t} \theta_{s, r_{i}+t}\right)+\lambda_{i, r_{i}} \theta_{i, r_{i}}+\mu
$$

where we put all the terms with $r_{s} \leq r_{i}$ in $\mu=\sum_{s=1}^{i} \sum_{t=1}^{r_{i}} \beta_{s, t} \theta_{s, t}$ with $\beta_{i, r_{i}}=0$. It's clear that $\mu \in \operatorname{span}\left\{£_{i} \cap £\right\}, \theta_{s, r_{i}+t} \in £ \backslash £_{i} \cap £$. In particular, we have $\mu\left(\bar{\tau}_{i, 1}\right)=0$ and $\mu\left(\bar{\tau}_{s, r_{s}-\left(r_{i}+t\right)+1}\right)=0$ for $1 \leq s \leq$ $i-1$ and $0 \leq t \leq r_{s}-r_{i}$. The following result provides another way to check equation (21). A partial result of the following proposition can be found in [16] for a triangular form without any proof.

Proposition 1: For $1 \leq i<j \leq m$, the following assertions are equivalent:

1) for $1 \leq k \leq r_{i}-r_{j}, d L_{f}^{r_{j}-1+k} h_{j} \in \operatorname{span}\left\{£_{i} \cap £\right\}$

2) for $1 \leq s \leq i-1,0 \leq t \leq r_{s}-r_{i}$ and $1 \leq k \leq r_{i}-r_{j}$,

$$
\left\{\begin{array}{l}
d L_{f}^{r_{j}-1+k} h_{j}\left(\bar{\tau}_{i, 1}\right)=0 \\
d L_{f}^{r_{j}-1+k} h_{j}\left(\bar{\tau}_{s, r_{s}-\left(r_{i}+t\right)+1}\right)=0
\end{array}\right.
$$

3) for $1 \leq s \leq i-1,0 \leq t \leq r_{s}-r_{i}$ and $1 \leq k \leq r_{i}-r_{j}$,

$$
\left\{\begin{array}{l}
d h_{j}\left(\bar{\tau}_{i, r_{j}+k}\right)=0 \\
d h_{j}\left(\bar{\tau}_{s, r_{s}-\left(r_{i}+t\right)+r_{j}+k}\right)=0
\end{array}\right.
$$

Proof. By definition of $\bar{\tau}_{j, s}$ given by (13-17) and definition of $£_{i}$ in (18), it is clear that the distribution given by the kernel of the co-distribution $\operatorname{span}\left\{£_{i} \cap £\right\}$ is spanned by $\bar{\tau}_{i, 1}$ and $\bar{\tau}_{s, r_{s}-\left(r_{i}+t\right)+1}$ for $1 \leq s \leq i-1$ and $0 \leq t \leq r_{s}-r_{i}$. Hence, from the expression (22), we can conclude that items 1) and 2) of Proposition 1 are equivalent.

In order to show that the items 1) and 2) are equivalent to the item 3) of Proposition 1, we would like firstly to prove that if $d L_{f}^{l} h_{j}\left(\bar{\tau}_{p, q}\right)=0$, then

$$
d L_{f}^{l+1} h_{j}\left(\bar{\tau}_{p, q}\right)=-d L_{f}^{l} h_{j}\left(\bar{\tau}_{p, q+1}\right)
$$

For this, let us recall that for a differential 1-form $v$, its differential $d v$ is a differential 2-form, defined by its evaluation on a pair of vector fields $X$ and $Y$ as follows:

$$
d v(X, Y)=L_{X} v(Y)-L_{Y} v(X)-v([X, Y]) .
$$

Now, in (24) let us set $v=d L_{f}^{l} h_{j}, X=f$ and $Y=\bar{\tau}_{p, q}$. As $v$ is an exact 1-form, then we have $0=L_{f} d L_{f}^{l} h_{j}\left(\bar{\tau}_{p, q}\right)-L_{\bar{\tau}_{p, q}} d L_{f}^{l} h_{j}(f)-$ $d L_{f}^{l} h_{j}\left(\left[f, \bar{\tau}_{p, q}\right]\right)$. Therefore, if $d L_{f}^{l} h_{j}\left(\bar{\tau}_{p, q}\right)=0$, the above equation becomes $0=-d L_{f}^{l+1} h_{j}\left(\bar{\tau}_{p, q}\right)+d L_{f}^{l} h_{j}\left(\bar{\tau}_{p, q+1}\right)$ which leads to the desired equation (23).

Consequently, as we have $d L_{f}^{l} h_{j}\left(\bar{\tau}_{p, q}\right)=0$ for $1 \leq l \leq r_{j}$ $2+k$, therefore, by induction we can obtain $d L_{f}^{r_{j}-1+k}\left(\bar{\tau}_{p, q}\right)=$ $d h_{j}\left(\bar{\tau}_{p, q+r_{j}+k-1}\right)$. Thus, by taking $\bar{\tau}_{p, q}=\bar{\tau}_{i, 1}$ or $\bar{\tau}_{s, r_{s}-\left(r_{i}+t\right)+1}$, with the knowledge of $d L_{f}^{l} h_{j}\left(\bar{\tau}_{p, q}\right)=0$ for $0 \leq l \leq r_{j}-2+k$, we proved the equivalence between item 2) and item 3) of Proposition 1.

\section{Remark 1:}

1) The item 3) of Proposition 1 is very useful because it uses only the objects that have already been computed (i.e., $d h_{j}, \bar{\tau}_{i, r_{j}+k}$ and $\left.\bar{\tau}_{s, r_{s}-\left(r_{i}+t\right)+r_{j}+k}\right)$. Thus, we do not need to compute highorder derivatives $d L_{f}^{r_{j}-1+k} h_{j}$ for $k \geq 1$;

2) If the item 3 ) of Proposition 1 is not fulfilled for some integers $s$ and $t$ for $1 \leq s \leq i-1$ and $0 \leq t \leq r_{s}-r_{i}$, then the $j^{\text {th }}$ output $y_{j}$ will be affected by the high-order derivative $y_{i}^{\left(r_{i}-\left(r_{j}+k\right)\right)}$ of the output $y_{i}$ or $y_{s}^{\left(r_{i}+t-\left(r_{j}+k\right)\right)}$ of the output $y_{s}$;

3) This shows that the condition (20) in Theorem 1 is fundamental for the case where the outputs are not affected by the others for the observer normal form (7-9) without the dynamics (8).

Example 1: In order to clarify the fact discussed above, consider the following dynamical system

$$
\left\{\begin{array}{l}
\dot{x}_{1}=0, \dot{x}_{2}=x_{1}, \dot{x}_{3}=x_{2}+x_{3} x_{4}, \dot{x}_{4}=x_{3} \\
\dot{x}_{5}=x_{5} x_{6}, \dot{x}_{6}=x_{5}, y_{1}=x_{4}, y_{2}=x_{3}+x_{6}
\end{array}\right.
$$

A straightforward calculation leads to $\theta_{1,1}=d x_{4}, \theta_{1,2}=d x_{3}$, $\theta_{1,3}=d x_{2}+x_{4} d x_{3}+x_{3} d x_{4}, \quad \theta_{1,4}=d x_{1}+d\left(x_{4} x_{2}+x_{3} x_{4}^{2}+x_{3}^{2}\right)$, $\theta_{2,1}=d x_{3}+d x_{6}$, and $\theta_{2,2}=d x_{5}+d x_{2}+x_{4} d x_{3}+x_{3} d x_{4}$. From equations (13-16) and (17) we obtain $\bar{\tau}_{1,1}=$ $\frac{\partial}{\partial x_{1}}, \bar{\tau}_{1,2}=\frac{\partial}{\partial x_{2}}, \quad \bar{\tau}_{1,3}=\frac{\partial}{\partial x_{3}}, \quad \bar{\tau}_{1,4}=\frac{\partial}{\partial x_{4}}+x_{4} \frac{\partial}{\partial x_{3}}, \quad \bar{\tau}_{2,1}=\frac{\partial}{\partial x_{5}}$, $\bar{\tau}_{2,2}=\frac{\partial}{\partial x_{6}}+x_{6} \frac{\partial}{\partial x_{5}}, £_{1}=\left\{\theta_{1,1}, \theta_{1,2}, \theta_{1,3}, \theta_{2,1}, \theta_{2,2}, d L_{f}^{2} h_{2}, d L_{f}^{3} h_{2}\right\}$, $£=\left\{\theta_{1,1}, \theta_{1,2}, \theta_{1,3}, \theta_{1,4}, \theta_{2,1}, \theta_{2,2}\right\}$, and $£_{1} \cap £=$ $\left\{\theta_{1,1}, \theta_{1,2}, \theta_{1,3}, \theta_{2,1}, \theta_{2,2}\right\}$.

It is clear that those vector fields commute, thus condition (19) of Theorem 1 is fulfilled. Thus, there exists a change of coordinates as $z_{1,1}=x_{1}, z_{1,2}=x_{2}, z_{1,3}=x_{3}-\frac{1}{2} x_{4}^{2}$ and $z_{1,4}=y_{1}=x_{4}$. However, condition (20) of Theorem 1 fails, since a straightforward calculation shows that $d L_{f}^{2} h_{2}=\theta_{1,4}+x_{6}\left(\theta_{2,2}-\theta_{1,3}\right)+x_{5}\left(\theta_{2,1}-\theta_{1,3}\right) \notin £_{1}-$ $\left(£_{1} \cap £\right)$. Indeed, we have $z_{2,1}=x_{5}-\frac{1}{2} x_{6}^{2}$ and $z_{2,2}=x_{6}=y_{2}-\dot{y}_{1}$. That means the output $z_{2,2}$ provided by this change of coordinates is affected by $\dot{y}_{1}$. Therefore it cannot be considered as a measured output for the obtained normal form. This can be explained by the fact that $d h_{2}\left(\bar{\tau}_{1,3}\right) \neq 0$ or $d L_{f}^{2} h_{2}\left(\bar{\tau}_{1,1}\right) \neq 0$. 
The second part of this section is to discuss some properties of the co-distribution $\Delta$ defined in (11) which will be used in the sequel to deduce the required change of coordinates.

Lemma 1: For (5-6), we have the following properties:

1) the co-distribution $\Delta$ defined in (11) and the distribution $\Delta^{\top}$ defined in (12) are involutive;

2) the co-distribution $\Delta$ defined in (11) and the distribution $\Delta^{\top}$ defined in (12) are both invariant with respect to the vector field $f$ of (5-6), i.e., $L_{f} v \in \Delta$ for any 1 -form $v \in \Delta$ and $[f, H] \in \Delta^{\top}$ for any vector field $H \in \Delta^{\top}$;

3) there exist $(n-r)$ vector fields, noted as $\left\{\tau_{r+1}, \cdots, \tau_{n}\right\}$, that span $\Delta^{\top}$ and commute, i.e., $\left[\tau_{i}, \tau_{j}\right]=0$ for $r+1 \leq i, j \leq n$.

Proof. The following will prove the above three results one by one. 1) It is clear that the co-distribution $\Delta$ defined in (11) is involutive since it is spanned by the exact forms $\theta_{j, k}$. Then for any $X, Y \in \Delta^{\top}$, and for $1 \leq i \leq m$ and $1 \leq j \leq r_{i}$, due to (24), we have $d \theta_{i, j}=0$, $\theta_{i, j}(Y)=\theta_{i, j}(X)=0$, which gives $\theta_{i, j}([X, Y])=0$. This is equivalent to $[X, Y] \in \Delta^{\top}$. Thus, the annihilator $\Delta^{\top}$ is also involutive.

2) Using the well-known Cartan's identity [32], we have $L_{f} \theta_{i, j}=$ $\iota_{f} d \theta_{i, j}+d \iota_{f} \theta_{i, j}=d \iota_{f} \theta_{i, j}$ because $d \theta_{i, j}=0$. On the other hand, $d l_{f} \theta_{i, j}=d \theta_{i, j}(f)=\theta_{i+1, j}$ for $1 \leq j \leq r_{i}-1$ and $d \theta_{i, r_{i}}(f)=$ $d L_{f}^{r_{i}+1} \theta_{i, r_{i}} \in \Delta$ due to the partial observability rank condition imposed by Assumption 1. Therefore, in any case we have $L_{f} \theta_{i, j} \in \Delta$.

Now, let $H \in \Delta^{\top}$. Since $\theta_{i, j}$ for $1 \leq i \leq m$ and $1 \leq j \leq r_{i}$ are closed, then thanks to (24) we have $\theta_{i, j}([f, H])=L_{f} \theta_{i, j}(H)-L_{H} \theta_{i, j}(f)=$ $-L_{H} \theta_{i, j}(f)$. Using again the Cartan's identity, we obtain $L_{H} \theta_{i, j}(f)=$ $i_{H} d \theta_{i, j}(f)$ because $i_{H} \theta_{i, j}(f)=0$ which is due to the fact that the interior product of a function by a vector field is equal to zero. As $d \theta_{i, j}(f)=L_{f} \theta_{i, j} \in \Delta^{\top}$, we obtain $\theta_{i, j}([f, H])=0$, thus $[f, H] \in \Delta^{\top}$. 3) As $\Delta^{\top}$ is involutive, it is well-known (see e.g. [33]) that there exists a basis $\left\{\tau_{r+1}, \cdots, \tau_{n}\right\}$ such that $\left[\tau_{i}, \tau_{j}\right]=0$ for all $r+1 \leq i, j \leq n$.

Lemma 2: Given a family of $\bar{\tau}_{i, j}$ deduced by equations (13-17) for $1 \leq i \leq m$ and $1 \leq j \leq r_{i}$, then for any $H \in \Delta^{\top}$ we have $\left[\bar{\tau}_{i, j}, H\right] \in \Delta^{\top}$, i.e., $\Delta^{\top}$ is invariant with respect to $\bar{\tau}_{i, j}$.

Proof. It should be noted that we need only to prove the result for $\bar{\tau}_{i, 1}$ with $1 \leq i \leq m$. The reason is that, according to the construction $\bar{\tau}_{i, j}=\left[\bar{\tau}_{i, j-1}, f\right]$ for $1 \leq i \leq m$ and $1 \leq j \leq r_{i}$, by using the well-known Jacobi's identity, we have

$$
\left[\bar{\tau}_{i, j}, H\right]=\left[\left[\bar{\tau}_{i, j-1}, f\right], H\right]=-\left[\left[H, \bar{\tau}_{i, j-1}\right], f\right]-\left[[f, H], \bar{\tau}_{i, j-1}\right]
$$

which belongs to $\Delta^{\top}$ if and only if $\left[\bar{\tau}_{i, j-1}, H\right] \in \Delta^{\top}$ since $[f, H] \in \Delta^{\top}$ thanks to second item of Lemma 1. Therefore, if we can prove the result stated in Lemma 2 for $\bar{\tau}_{i, 1}$ with $1 \leq i \leq m$, then it will be satisfied as well for $\bar{\tau}_{i, j}$ for $1 \leq i \leq m$ and $2 \leq j \leq r_{i}$.

To achieve such a goal, we should prove $\theta_{s, t}\left(\left[\bar{\tau}_{i, 1}, H\right]\right)=0$ for $1 \leq$ $i \leq m, 1 \leq s \leq m, 1 \leq t \leq r_{s}$, and this is equivalent to $\left[\bar{\tau}_{i, 1}, H\right] \in \Delta^{\top}$.

As $\theta_{s, t}$ for $1 \leq s \leq m$ and $1 \leq t \leq r_{s}$ are closed and $\theta_{s, t}(H)=0$ for any $H \in \Delta^{\top}$, then from formula (24) we have $\theta_{s, t}\left(\left[\bar{\tau}_{i, 1}, H\right]\right)=$ $L_{H} \theta_{s, t}\left(\bar{\tau}_{i, 1}\right)$ for which the following two different cases should be distinguished.

Case 1: $i=1$

According to (13-16), we know that $\theta_{s, t}\left(\bar{\tau}_{1,1}\right)$ is a constant (either 0 or 1 for different indices), therefore its Lie derivative is 0 . Thus $\theta_{s, t}\left(\left[\bar{\tau}_{1,1}, H\right]\right)=L_{H} \theta_{s, t}\left(\bar{\tau}_{1,1}\right)=0$, which implies that $\left[\bar{\tau}_{1,1}, H\right] \in \Delta^{\top}$. Case 2: $2 \leq i \leq m$

For $i \neq 1$, the function $\theta_{s, t}\left(\bar{\tau}_{i, 1}\right)$ is not necessarily constant for all $\theta_{s, t}$. In what follows, we will prove the result for $i=2$, and the same argument can be used to prove the result for $3 \leq i \leq m$.

According to (13-16), it can be seen that $\theta_{s, t}\left(\bar{\tau}_{2,1}\right)=0$ for $2 \leq s \leq m$ and $1 \leq t \leq r_{s}$, except when $s=2$ and $t=r_{2}$ for which $\theta_{2, r_{2}}\left(\bar{\tau}_{2,1}\right)=1$. On the other hand we have $\theta_{1, t}\left(\bar{\tau}_{2,1}\right)=0$ for $1 \leq t \leq r_{2}$. Consequently, for all $\theta_{s, t}$, we have $\theta_{s, t}\left(\left[\bar{\tau}_{2,1}, H\right]\right)=0$ for any $H \in \Delta^{\top}$.
It remains us to prove that the result is true as well for $\theta_{1, r_{2}+1}, \cdots, \theta_{1, r_{1}}$. As we have proved in the above that it is always true if $\theta_{1, k}\left(\bar{\tau}_{2,1}\right)$ for $r_{2}+1 \leq k \leq r_{1}$ is constant. In the following, we will show that it is true as well even if $\theta_{1, k}\left(\bar{\tau}_{2,1}\right)$ is not constant.

For this, for any $\theta_{1, k}\left(\bar{\tau}_{2,1}\right)=\lambda$ which is not constant, we can rewrite it as follows: $\theta_{1, k}=\lambda \theta_{2, r_{2}}+v$ where $v$ is 1-form such that $v\left(\bar{\tau}_{2,1}\right)=0$, since $\theta_{1, k}$ and $\theta_{2, r_{2}}$ belong to $\Delta$ which implies $v \in \Delta$. Therefore, we have

$$
v=\sum q_{i, j} \theta_{i, j}
$$

which is a combination of some $\theta_{i, j}$ that vanish on $\tau_{2,1}$ and $q_{i, j}$ are functions.

As $\theta_{2, r_{2}}\left(\left[\bar{\tau}_{2,1}, H\right]\right)=0$ because of the formula (24), due to the fact that $\theta_{2, r_{2}}\left(\bar{\tau}_{2,1}\right)=\theta_{2, r_{2}}(H)=0$, then $\theta_{2, r_{2}}\left(\left[\bar{\tau}_{2,1}, H\right]\right)=0$ is equivalent to $v\left(\left[\bar{\tau}_{2,1}, H\right]\right)=0$. To show this, consider again the formula (24) for $v: d v\left(\bar{\tau}_{2,1}, H\right)=L_{\bar{\tau}_{2,1}} v(H)-L_{H} v\left(\bar{\tau}_{2,1}\right)-v\left(\left[\bar{\tau}_{2,1}, H\right]\right)$. As $v(H)=v\left(\bar{\tau}_{2,1}\right)=0$, this equation becomes $d v\left(\bar{\tau}_{2,1}, H\right)=$ $-v\left(\left[\bar{\tau}_{2,1}, H\right]\right)$. On the other hand, the differentiation of (25) gives $d v=\sum d q_{i, j} \wedge \theta_{i, j}+\sum q_{i, j} d \theta_{i, j}=\sum d q_{i, j} \wedge \theta_{i, j}$ since $d \theta_{i, j}=0$. Hence, $d v\left(\bar{\tau}_{2,1}, H\right)=\sum d q_{i, j}\left(\bar{\tau}_{2,1}\right) \theta_{i, j}(H)-d q_{i, j}(H) \theta_{i, j}\left(\bar{\tau}_{2,1}\right)=0$ since $\theta_{i, j}(H)=\theta_{i, j}\left(\bar{\tau}_{2,1}\right)=0$. Finally, we obtain $\theta_{1, k}\left(\left[\bar{\tau}_{2,1}, H\right]\right)=$ $v\left(\left[\bar{\tau}_{2,1}, H\right]\right)=0$, and we proved $\left[\bar{\tau}_{2,1}, H\right] \in \Delta^{\top}$ for any $H \in \Delta^{\top}$.

The above lemma enables us to state the following result.

Corollary 1: Given a family of $\bar{\tau}_{i, j}$ deduced by equations (13-17) for $1 \leq i \leq m$ and $1 \leq j \leq r_{i}$, then we have

$$
\left[\bar{\tau}_{i, k}+H_{i}, \bar{\tau}_{j, s}+H_{j}\right]=\left[\bar{\tau}_{i, k}, \bar{\tau}_{j, s}\right] \text { modulo } \Delta^{\top}
$$

for any $H_{i} \in \Delta^{\top}$ and $H_{j} \in \Delta^{\top}$.

Proof. Given a family of $\bar{\tau}_{i, j}$ deduced by equations (13-17) for $1 \leq$ $i \leq m$ and $1 \leq j \leq r_{i}$, a straight calculation yields

$$
\left[\bar{\tau}_{i, k}+H_{i}, \bar{\tau}_{j, s}+H_{j}\right]=\left[\bar{\tau}_{i, k}, \bar{\tau}_{j, s}\right]+\left[\bar{\tau}_{i, k}, H_{j}\right]+\left[H_{i}, \bar{\tau}_{j, s}\right]+\left[H_{i}, H_{j}\right]
$$

We know from Lemma 1 that $\Delta^{\top}$ is involutive, thus $\left[H_{i}, H_{j}\right] \in \Delta^{\top}$. From Lemma 2 we deduced that $\left[\bar{\tau}_{i, k}, H_{j}\right]$ and $\left[H_{i}, \bar{\tau}_{j, s}\right]$ both belong to $\Delta^{\top}$, thus we proved the result stated in Corollary 1 .

\section{MAIN RESUlT}

This section deals with the geometric condition that guarantees the existence of a change of coordinates transforming system (5-6) into the proposed observer normal form (7-8). Before stating the main result of this work, we need the following lemma.

Lemma 3: For a family of $\bar{\tau}_{1, k}$ satisfying (13-16) and the associated $\bar{\tau}_{i, k}$ for $2 \leq k \leq r_{i}$ deduced from (17), there exists a family of vector fields $\tau_{i, k}=\bar{\tau}_{i, k}$ modulo $\Delta^{\perp}$ such that $\left[\tau_{i, k}, \tau_{j, s}\right]=0$ for $1 \leq k \leq r_{i}$ and $1 \leq s \leq r_{j}$, if and only if $\left[\bar{\tau}_{i, k}, \bar{\tau}_{j, s}\right] \in \Delta^{\perp}$.

Proof. Necessity: Suppose that there exist $H_{i, k} \in \Delta^{\perp}$ and $H_{j, s} \in \Delta^{\perp}$, which determine $\tau_{i, k}=\bar{\tau}_{i, k}+H_{i, k}$ and $\tau_{j, s}=\bar{\tau}_{j, s}+H_{j, s}$ for $1 \leq k \leq r_{i}$ and $1 \leq s \leq r_{j}$ such that $\left[\tau_{i, k}, \tau_{j, s}\right]=0$, then we have $0=\left[\tau_{i, k}, \tau_{j, s}\right]=$ $\left[\bar{\tau}_{i, k}, \bar{\tau}_{j, s}\right]$ modulo $\Delta^{\perp}$, which implies that $\left[\bar{\tau}_{i, k}, \bar{\tau}_{j, s}\right] \in \Delta^{\perp}$.

Sufficiency: Assume that for one choice of the family $\bar{\tau}_{1, k}$ satisfying (13-16), we have $\left[\bar{\tau}_{i, k}, \bar{\tau}_{j, s}\right] \in \Delta^{\perp}$ for $1 \leq k \leq r_{i}$ and $1 \leq s \leq r_{j}$ defined in (17). Then we need to prove that there exist $H_{i, j} \in \Delta^{\perp}, H_{j, s} \in \Delta^{\perp}$ for $1 \leq k \leq r_{i}$ and $1 \leq s \leq r_{j}$ such that $\left[\tau_{i, k}, \tau_{j, s}\right]=0$ where $\tau_{i, k}=\bar{\tau}_{i, k}+H_{i, k}$ and $\tau_{j, s}=\bar{\tau}_{j, s}+H_{j, s}$.

Let $\left\{\tau_{r+1}, \cdots, \tau_{n}\right\}$ be $(n-r)$ determined commutative basis of $\Delta^{\perp}$, it is well-known (see [33]) that there exist $r$ commutative vector fields, noted as $\left\{X_{1}, \cdots, X_{r}\right\}$, such that they commute with $\left\{\tau_{r+1}, \cdots, \tau_{n}\right\}$, thus form together a basis of the tangent fiber bundle $T \mathscr{X}$ of $\mathscr{X}$.

Therefore, we can rewrite $\bar{\tau}_{i, k}$ as: $\bar{\tau}_{i, k}=\sum_{l=1}^{r} \gamma_{l}^{i, k}(x) X_{l}+$ $\sum_{l=r+1}^{n} \mu_{l}^{i, k}(x) \tau_{l}$ where functions $\gamma_{l}^{i, k}(x)$ are constant on the leaves of $\Delta^{\perp}$ thus $L_{\tau_{i}} \gamma_{l}^{i, k}=0$ because of the invariance of $\Delta^{\perp}$ by 
means of $\bar{\tau}_{i, k}$ thanks to Lemma $2:\left[\bar{\tau}_{i, j}, \tau_{i}\right] \in \Delta^{\perp}$. By choosing $H_{i, k}=-\sum_{l=r+1}^{n} \mu_{l}^{i, k}(x) \tau_{l} \in \Delta^{\perp}$, then we have: $\tau_{i, j}=\bar{\tau}_{i, k}+$ $H_{i, k}=\sum_{l=1}^{r} \gamma_{l}^{i, k}(x) X_{l}$. For the same reason, there exists $H_{j, s}=$ $\sum_{l=r+1}^{n} \kappa_{l}^{j, s}(x) \tau_{l} \in \Delta^{\perp}$ yielding: $\tau_{j, s}=\bar{\tau}_{j, s}+H_{j, s}=\sum_{l=1}^{r} \alpha_{l}^{j, s}(x) X_{l}$. Moreover, for $1 \leq j \leq r_{i}$ and $1 \leq s \leq r_{t}$, due to the invariance of $\Delta^{\perp}$ by means of $\bar{\tau}_{i, j}$, we have

$$
\left[\bar{\tau}_{i, k}, \bar{\tau}_{j, s}\right]=\left[\tau_{i, k}, \tau_{j, s}\right] \text { modulo } \Delta^{\perp}
$$

As $\left[X_{k}, X_{s}\right]=0$ for $1 \leq k, s \leq r$ and the fact that functions $\gamma_{l}^{i, k}$ and $\alpha_{l}^{j, s}$ are constant on the leaves of $\Delta^{\perp}$, then (27) becomes $\left[\bar{\tau}_{i, k}, \bar{\tau}_{j, s}\right]=$ $\left[\sum_{l, m=1}^{r} \gamma_{l}^{i, k} L_{X_{l}} \alpha_{m}^{j, s} X_{m}-\sum_{m, l=1}^{r} \alpha_{l}^{j, s} L_{X_{m}} \gamma_{l}^{i, k} X_{l}\right] \operatorname{modulo} \Delta^{\perp}$.

Thus, if $\left[\bar{\tau}_{i, k}, \bar{\tau}_{j, s}\right] \in \Delta^{\perp}$, then there exist $H_{i, k} \in$ $\Delta^{\perp} \quad$ and $\quad H_{j, s} \in \Delta^{\perp}$ defined above, such that $\left[\tau_{i, k}, \tau_{j, s}\right]=\sum_{l, m=1}^{r} \gamma_{l}^{i, k} L_{X_{l}} \alpha_{m}^{j, s} X_{m}-\sum_{m, l=1}^{r} \alpha_{l}^{j, s} L_{X_{m}} \gamma_{l}^{i, j} X_{l}=0$ which is due to the fact that $X_{k} \notin \Delta^{\perp}$.

Based on the preliminary results presented in Section III and in the above Lemma 3, we are now ready to state our main theorem.

Theorem 2: There exists a local change of coordinates $\left(z^{T}, \eta^{T}\right)^{T}=$ $\phi(x)$ which transforms system (5-6) into the observer normal form (7-8) if and only if the following conditions are satisfied:

1) there exists a family of vector fields $\bar{\tau}_{i, 1}$ for $1 \leq i \leq m$ which are solutions of algebraic equations (13-16), such that we have

$$
\left[\bar{\tau}_{i, k}, \bar{\tau}_{j, s}\right] \in \Delta^{\top}
$$

where $\bar{\tau}_{i, k}$ and $\bar{\tau}_{j, s}$ are defined by (17) for all $1 \leq i, j \leq m, 1 \leq$ $k \leq r_{i}$ and $1 \leq s \leq r_{j}$

2) for all $1 \leq i \leq m, 1 \leq k \leq r_{i}$ we have

$$
\left[\bar{\tau}_{i, k}, \tau_{l}\right] \in \Delta
$$

where the vector fields $\tau_{l}$, for $r+1 \leq l \leq n$, were deduced in the item 3) of Lemma 1 ;

3) for $1<i<j \leq m$ and $r_{i}>r_{j}$ we have

$$
d h_{j}\left(\bar{\tau}_{i, r_{j}+k}\right)=0 \text { and } d h_{j}\left(\bar{\tau}_{s, r_{s}-\left(r_{i}+t\right)+r_{j}+k}\right)=0,
$$

with $1 \leq k \leq r_{i}-r_{j}, 1 \leq s \leq i-1$ and $0 \leq t \leq r_{s}-r_{i}$

Proof. Necessity: Suppose that there exists a local change of coordinates $\left(z^{T}, \eta^{T}\right)^{T}=\phi(x)$ which transforms (5-6) into (7-8). Note $\omega=d \phi(x)$, then $\omega$ is the pullback of $d z$ and $d \eta$ for (7-8). Therefore, we need only to check the necessity for (7-8). It is clear that, for (7-8), there exist $\bar{\tau}_{i, k}=\frac{\partial}{\partial z_{i, k}}$ for $1 \leq i \leq m$ and $1 \leq k \leq r_{i}$ and $\tau_{r+j}=\frac{\partial}{\partial \eta_{j}}$ for $1 \leq j \leq n-r$ such that all conditions in Theorem 2 are satisfied for (7-8). Then, according to the property of pullback, we can conclude that those conditions are necessary for (5-6).

Sufficiency: Suppose that all conditions in Theorem 2 are satisfied. Then we can construct the following multi-valued matrix $\Lambda$ :

$$
\Lambda=\left(\begin{array}{ccccc}
\theta_{1,1}\left(\bar{\tau}_{1,1}\right) & \cdots & \theta_{1,1}\left(\bar{\tau}_{1, r_{1}}\right) & \cdots & \theta_{1,1}\left(\bar{\tau}_{m, r_{m}}\right) \\
\vdots & \ddots & \vdots & \ddots & \vdots \\
\theta_{1, r_{1}}\left(\bar{\tau}_{1,1}\right) & \cdots & \theta_{1, r_{1}}\left(\bar{\tau}_{1, r_{1}}\right) & \cdots & \theta_{1, r_{1}}\left(\bar{\tau}_{m, r_{m}}\right) \\
\theta_{2,1}\left(\bar{\tau}_{1,1}\right) & \cdots & \theta_{2,1}\left(\bar{\tau}_{1, r_{1}}\right) & \cdots & \theta_{2,1}\left(\bar{\tau}_{m, r_{m}}\right) \\
\vdots & \ddots & \vdots & \ddots & \vdots \\
\theta_{m, r_{m}}\left(\bar{\tau}_{1,1}\right) & \cdots & \theta_{m, r_{m}}\left(\bar{\tau}_{1, r_{1}}\right) & \cdots & \theta_{m, r_{m}}\left(\bar{\tau}_{m, r_{m}}\right)
\end{array}\right) .
$$

Let $u$ set $\theta=\left(\theta_{1,1}, \cdots, \theta_{1, r_{1}}, \theta_{2,1}, \cdots, \theta_{m, r_{m}}\right)^{T}$ and $\bar{\tau}=$ $\left(\begin{array}{lllll}\bar{\tau}_{1,1} & \cdots & \bar{\tau}_{1, r_{1}} & \bar{\tau}_{2,1} \cdots & \bar{\tau}_{m, r_{m}}\end{array}\right)$. The 1 -forms $\theta_{i, j}$ can be seen as co-vectors (row vectors) and $\bar{\tau}_{i, j}$ are column vectors. Therefore, $\theta$ and $\bar{\tau}$ are matrices of type $r \times n$ and $n \times r$, respectively. As the 1 -forms $\theta_{i j}$ and the vectors $\bar{\tau}_{i, j}$ are both independent, then $\theta$ and $\bar{\tau}$ are both of rank $r$. Recall that $\Delta^{T}=\operatorname{ker} \theta$, and we have $\Delta^{T} \bigcap \operatorname{span}\left\{\bar{\tau}_{i, j}\right\}=0$, then we deduce that $\Lambda=\theta \times \bar{\tau}$ is a $r \times r$ invertible matrix. Hence, we can define $r$ differential 1 -forms as follows:

$$
\omega=\left(\omega_{1,1}, \cdots, \omega_{1, r_{1}}, \omega_{2,1}, \cdots, \omega_{m, r_{m}}\right)^{T}=\Lambda^{-1} \theta
$$

From the above formula, we see that 1 -forms $\omega_{i, j}$ are linear combinations of 1 -forms $\theta_{i, j}$. Thus they span the same co-distribution $\Delta$ and have the annihilator as $\Delta^{\top}$. Therefore, they are well defined from any family $\bar{\tau}_{i, 1}$ modulo $\Delta^{\top}$. By the definition we have $\omega_{i, j}\left(\bar{\tau}_{s, t}\right)=\delta_{i}^{s} \delta_{j}^{t}$ where $\delta_{i}^{s}$ is the Kronecker delta, therefore if the condition (28) of Theorem 2 is fulfilled, then the co-distribution spanned by $\omega_{i, j}$ is integrable. From the dual version of the result (see Theorem 2.42 and Corollary 2.43 and their proofs on page 57 of [33]), there always exist $\omega_{k}$ for $1 \leq k \leq n-r$ that complete this basis. In other words, we can find $n-r$ differential 1 -forms $\omega_{j}$ for $1 \leq j \leq n-r$ such that

- $\omega_{j}\left(\tau_{k}\right)=\delta_{k}^{j}$ for $r+1 \leq k, j \leq n$;

- $\omega_{j}\left(\bar{\tau}_{s, t}\right)=0$ thus the annihilator of these 1 -forms is spanned by $\bar{\tau}_{s, t}$.

Note that, by using formula (24), the above 1- forms $\omega_{j}$ together with $\omega_{i, k}$ are independent and closed. Then, according to the construction procedure, we have $\omega_{j}\left(\tau_{r+j}\right)=\frac{\partial}{\partial \eta_{j}}$ for $1 \leq j \leq n-r$ and $\omega_{i, k}\left(\bar{\tau}_{i, k}\right)=$ $\frac{\partial}{\partial z_{i, k}}$. Therefore, thanks to Poincaré's Lemma, there exists a change of coordinates $\phi(x)=\left(z^{T}, \eta^{T}\right)^{T}$ such that the differential of $\phi$ is $\phi_{*}=\omega$.

Let us show how the vector field $f$ in the dynamical system (5-6) is transformed by $\phi$. To do so, we compute $\frac{\partial}{\partial \eta_{j}} \phi_{*}(f)$, and we obtain $\frac{\partial}{\partial \eta_{j}} \phi_{*}(f)=\left[\frac{\partial}{\partial \eta_{j}}, \phi_{*}(f)\right]$. Then we have $\frac{\partial}{\partial \eta_{j}} \phi_{*}(f)=\phi_{*}\left(\left[\tau_{r+j}, f\right]\right) \in \phi_{*}\left(\Delta^{\top}\right) \quad$ because $\tau_{r+j} \in \Delta^{\top}$ and $\Delta^{\top}$ is $f$-invariant by Lemma 1 . Therefore $\frac{\partial}{\partial \eta_{j}} \phi_{*}(f) \in \operatorname{span}\left\{\frac{\partial}{\partial \eta_{k}}\right.$, for $\left.1 \leq k \leq n-r\right\}$.

Now we compute $\frac{\partial}{\partial z_{i, j}} \phi_{*}(f)$ for $1 \leq i \leq m$ and $1 \leq j \leq r_{i}-1$, and we obtain $\frac{\partial}{\partial z_{i, j}} \phi_{*}(f)=\phi_{*}\left(\left[\bar{\tau}_{i, j}, f\right]\right)=\phi_{*}\left(\bar{\tau}_{i, j+1}+\right.$ $H)=\phi_{*}\left(\bar{\tau}_{i, j+1}\right)+\phi_{*}(H)$ where $H \in \Delta^{\top}$ which gives $\frac{\partial}{\partial z_{i, j}} \phi_{*}(f)=\frac{\partial}{\partial z_{i, j+1}}$ modulo $\Delta^{\top}$. Therefore, by integration we have $\phi_{*}(f)=\sum_{j=1}^{m}\left(\sum_{k=1}^{r_{j}-1} z_{j, k} \frac{\partial}{\partial z_{j, k+1}}\right)+\beta\left(z_{r_{1}}, \cdots, z_{r_{m}}\right)$ modulo $\Delta^{\top}$. It remains to find the relation between $\left(z_{r_{1}}, \cdots, z_{r_{m}}\right)$ and the outputs $y_{1}, \cdots, y_{m}$. For this, it should be noted that from the equation (30), together with (16) for $1 \leq i, j \leq m$ and $r_{i}>r_{j}+1$, we have $d L_{f}^{k} h_{j}\left(\bar{\tau}_{i, 1}\right)=0$ for $0 \leq k \leq r_{i}-1$.

Now, using formula (24) for $v=d L_{f}^{k} h_{j}, X=f$ and $Y=\bar{\tau}_{i, 1}$ we obtain $0=L_{f} d L_{f}^{k} h_{j}\left(\bar{\tau}_{i, 1}\right)-L_{\tau_{i, 1}} d L_{f}^{k} h_{j}(f)-d L_{f}^{k} h_{j}\left[f, \bar{\tau}_{i, 1}\right]$.

If $d L_{f}^{k} h_{j}\left(\bar{\tau}_{i, 1}\right)=0$, then we obtain $d L_{f}^{k+1} h_{j}\left(\bar{\tau}_{i, 1}\right)=d L_{f}^{k} h_{j}\left(\bar{\tau}_{i, 2}\right)$. Therefore, by induction we deduce that $d h_{j}\left(\bar{\tau}_{i, r_{i}-1}\right)=\cdots=$ $d h_{j}\left(\bar{\tau}_{i, 1}\right)=0$..

Thus the output $h_{j}$ does not depend on $\dot{y}_{i}, \cdots, y_{i}^{\left(r_{i}-1\right)}$. However, $d h_{j}\left(\bar{\tau}_{i, r_{i}}\right)$ may not be equal to 0 . Therefore by integration we obtain $y_{j}=z_{r_{j}}+\varphi_{j}\left(z_{r_{1}}, \cdots, z_{r_{j-1}}\right) .$. As $z_{r_{1}}=y_{1}, z_{r_{2}}=y_{2}+\varphi\left(y_{1}\right)$ and so forth $z_{r_{m}}=y_{m}+\varphi\left(y_{1}, \cdots, y_{m-1}\right)$, finally we obtain (9).

The following statements can be made by applying the results of Lemma 2 and Corollary 1.

Remark 2:

1) The following two items are equivalent

- The items 1), 2) and 3) of Theorem 2 are fulfilled by at least one family of vector fields $\bar{\tau}_{i, j}$;

- The items 1), 2) and 3) of Theorem 2 are fulfilled for the family of vector fields $\tau_{i, j}$ obtained from any $\bar{\tau}_{i, j}$ by removing the components in $\tau_{l}$ directions. Especially, this family fulfills the following commutativity conditions $\left[\tau_{i, k}, \tau_{j, s}\right]=0$ and $\left[\tau_{i, k}, \tau_{l}\right]=0$. 
2) If the dynamical system (5-6) is fully observable, i.e., $\operatorname{dim} \Delta=$ $r=n$, then $\Delta^{\top}=0$. Therefore, condition (28) of Theorem 2 becomes the same as condition (19) of Theorem 1, condition (29) is always satisfied and thanks to the Proposition 1, equation (30) is equivalent to equation (20). Thus, in this sense, we can say that Theorem 2 is a generalization of Theorem 1 .

Let show an example to highlight the first two items in Remark 2.

Example 2: Consider the following dynamical system

$$
\left\{\begin{array}{l}
\dot{x}_{1}=x_{3} x_{1}, \dot{x}_{2}=x_{1}, \dot{x}_{3}=-x_{3}+x_{3} x_{2}^{2} \\
\dot{x}_{4}=-x_{4}+x_{1} x_{3}^{2}+x_{4}^{2}, y_{1}=x_{2}, y_{2}=x_{3}
\end{array}\right.
$$

A straightforward calculation leads to $\theta_{1,1}=d x_{2}, \theta_{1,2}=d x_{1}$ and $\theta_{2,1}=d x_{3}$. A solution of (13-16) can be chosen as $\bar{\tau}_{1,1}=\frac{\partial}{\partial x_{1}}, \bar{\tau}_{1,2}=$ $\frac{\partial}{\partial x_{2}}+x_{3} \frac{\partial}{\partial x_{1}}+x_{3}^{2} \frac{\partial}{\partial x_{4}}$ and $\bar{\tau}_{1,3}=\frac{\partial}{\partial x_{3}}$. It's clear that $\Delta=\operatorname{span}\left\{\frac{\partial}{\partial x_{4}}\right\}$. Therefore, we obtain $\left[\bar{\tau}_{1,2}, \bar{\tau}_{1,3}\right]=-\frac{\partial}{\partial x_{1}}-2 x_{3} \frac{\partial}{\partial x_{4}} \notin \Delta^{\top}$ because of the presence of the direction $\frac{\partial}{\partial x_{1}}$. However, if we choose $\bar{\tau}_{1,3}=$ $\frac{\partial}{\partial x_{3}}+x_{2} \frac{\partial}{\partial x_{1}}$, then the Lie bracket becomes $\left[\bar{\tau}_{1,2}, \bar{\tau}_{1,3}\right]=-2 x_{3} \frac{\partial}{\partial x_{4}}$ which belongs to $\Delta^{\top}$. As $\tau_{4}=\frac{\partial}{\partial x_{4}}$, the condition (29) in Theorem 2 is also fulfilled. The second item of Remark 2 means that we can choose $\bar{\tau}_{1,2}=\frac{\partial}{\partial x_{2}}+x_{3} \frac{\partial}{\partial x_{1}}$ modulo $\Delta^{\top}$. The last condition in Theorem 2 is obviously satisfied, because in this case we have $r_{1}=r_{2}+1$. The above discussion is in accordance with the fact that if we take the following change of coordinates $z_{1}=x_{1}-x_{3} x_{2}$ and keep other variables unchanged, we can obtain the desired normal form.

Theorem 2 is for system (5-6) which does not contain any input. The extension of this result to treat systems with inputs is presented in the following corollary.

Corollary 2: Consider

$$
\begin{aligned}
& \dot{x}=f(x)+\sum_{k=1}^{s} g_{k}(x) u_{k} \\
& y=h(x)
\end{aligned}
$$

where $u_{k} \in \mathbb{R}$ for $1 \leq k \leq s$ is input. Assume that the pair $(f, h)$ fulfills the conditions in Theorem 2. Then the above system can be transformed into

$$
\begin{aligned}
\dot{z}_{i} & =A_{i} z_{i}+\beta_{i}(\bar{y})+\sum_{k=1}^{s} \gamma_{1, k}(\bar{y}) u_{k} \\
\dot{\eta} & =\xi(z, \eta)+\sum_{k=1}^{s} \gamma_{2, k}(\bar{y}) u_{k} \\
\bar{y}_{i} & =C_{i} z_{i}
\end{aligned}
$$

if and only if the following conditions are satisfied:

- $\left[\bar{\tau}_{i, j}, g_{k}\right] \in \Delta$ for all $1 \leq i \leq m, 1 \leq j \leq r_{i}-1$ and $1 \leq k \leq s$

- $\left[\tau_{l}, g_{k}\right] \in \Delta$ for all $r+1 \leq l \leq n$ and $1 \leq k \leq s$

where $\bar{\tau}_{i, j}$ and $\tau_{l}$ are given in Theorem 2 .

Proof. The proof of this corollary is quite similar to that of Theorem 2. The following just gives a sketch of the proof.

Since the pair $(f, h)$ of (32) satisfies the conditions in Theorem 2 , then there exists a diffeomorphism $\phi$ such that the vector field $f$ of (32) can be transformed as $\phi_{*}(f)=\left[\begin{array}{c}A_{i} z_{i}+\beta_{i}(\bar{y}) \\ \xi(z, \eta)\end{array}\right]$. Now let us see how $\phi_{*}$ transforms the vector fields $g_{k}$ for $1 \leq k \leq s$. For this, we need to compute $\frac{\partial}{\partial z_{i, j}} \phi_{*}\left(g_{k}\right)$ and $\frac{\partial}{\partial z_{l}} \phi_{*}\left(g_{k}\right)$ for $1 \leq i \leq m$, $1 \leq j \leq r_{i}-1, r+1 \leq l \leq n$ and $1 \leq k \leq s$. The stated two conditions in Corollary 2 are then equivalent to $\frac{\partial}{\partial z_{i, j}} \phi_{*}\left(g_{k}\right)=\phi_{*}\left[\bar{\tau}_{i, j}, g_{k}\right] \in \Delta$ and $\frac{\partial}{\partial z_{l}} \phi_{*}\left(g_{k}\right)=\phi_{*}\left[\tau_{l}, g_{k}\right] \in \Delta$. Hence, we get the stated result by simply following the same arguments as those in Theorem 2 for $\phi_{*}(f)$.

Given a multi-output nonlinear dynamical system of the form (5$6)$, the following summarizes the complete procedure when applying the proposed method:

Step 1: Determine the observability indices $r_{i}$ for $1 \leq i \leq m$ and compute the associated observability 1 -forms $\theta_{i, j}$ for $1 \leq i \leq m$ and $1 \leq j \leq r_{i}$;

Step 2: Determine $\Delta, \Delta^{\perp}$ and calculate a commutative basis $\left\{\tau_{r+1}, \cdots, \tau_{n}\right\}$ that spans $\Delta^{\perp}$;
Step 3: Choose any solution $\bar{\tau}_{i, 1}$ of (13-16) for $1 \leq i \leq m$, and eliminate all its terms modulo $\Delta^{\perp}$ which yields $\tau_{i, 1}$;

Step 4: Iteratively, for $2 \leq j \leq r_{i}$, compute $\left[\tau_{i, j}, f\right]$ and eliminate all its terms modulo $\Delta^{\perp}$ which yields $\tau_{i, j}$;

Step 5: Calculate the multi-valued matrix $\Lambda$ and the associated $r$ differential 1 -forms $\omega$;

Step 6: Integrating $\omega$ yields $z=\phi_{1}(x)$, and then we can choose $\eta=\phi_{2}(x)$, complementary to $\phi_{1}(x)$ such that $\phi(x)=$ $\left[\phi_{1}^{T}(x), \phi_{2}^{T}(x)\right]^{T}$ forms a change of coordinates;

Step 7: For (5-6), applying $\phi(x)=\left(z^{T}, \eta^{T}\right)^{T}$ to obtain the form (7$9)$, and design an observer of form (10).

\section{EXAMPLE}

In order to highlight the proposed result, let us consider the following nonlinear system:

$$
\left\{\begin{array}{l}
\dot{x}_{1}=x_{2} x_{5}+x_{3} x_{4}-x_{3}^{3}+x_{3} x_{5}, \dot{x}_{2}=x_{1}, \dot{x}_{3}=x_{2} \\
\dot{x}_{4}=-2 x_{3} x_{4}+2 x_{3}^{3}-2 x_{2} x_{5}, \dot{x}_{5}=x_{4}-x_{3}^{2} \\
\dot{x}_{6}=-x_{6}+x_{2} x_{5}, y_{1}=x_{3}, y_{2}=x_{5}
\end{array}\right.
$$

In the literature, for a general nonlinear system, most of existing methods of observer design need to impose some additional assumptions on the nonlinear terms, such as global Lipschitz condition [34], or similar norm-inequalities [35]. Concerning the above example which contains the quadratic and cubic terms, it can be easily checked that those assumptions are not satisfied. Consequently, these techniques cannot be applied to design observer for the studied example. The following shows, via the diffeomorphism, a simple Luenberger-like observer can be designed for this example.

Step 1-2: A straightforward calculation gives $\theta_{1,1}=d x_{3}, \theta_{1,2}=d x_{2}$, $\theta_{1,3}=d x_{1}, \theta_{2,1}=d x_{5}$ and $\theta_{2,2}=d x_{4}-2 x_{3} d x_{3}$, we can see that those 1 -forms are independent. Thus, $\Delta=\operatorname{span}\left\{\theta_{1,1}, \theta_{1,2}, \theta_{1,3}, \theta_{2,1}, \theta_{2,2}\right\}$ is of rank 5 , thus we have observability indices $r_{1}=3$ and $r_{2}=2$, and we have $\Delta^{T}=\operatorname{span}\left\{\tau_{6}\right\}$ where $\tau_{6}=\frac{\partial}{\partial x_{6}}$.

Step 3-4: Now, let us take the following one solution of (13-16): $\bar{\tau}_{1,1}=\frac{\partial}{\partial x_{1}}$ and $\bar{\tau}_{2,1}=\frac{\partial}{\partial x_{4}}$, and compute the other vector fields by using (17): $\bar{\tau}_{1,2}=\frac{\partial}{\partial x_{2}}, \bar{\tau}_{1,3}=\frac{\partial}{\partial x_{3}}+x_{5} \frac{\partial}{\partial x_{1}}-2 x_{5} \frac{\partial}{\partial x_{4}}+x_{5} \frac{\partial}{\partial x_{6}}, \bar{\tau}_{2,2}=$ $\frac{\partial}{\partial x_{5}}+x_{3} \frac{\partial}{\partial x_{1}}-2 x_{3} \frac{\partial}{\partial x_{4}}$ It is easy to check that $\left[\bar{\tau}_{i, j}, \bar{\tau}_{k, s}\right]=0$ except for $\bar{\tau}_{1,3}$ with $\bar{\tau}_{2,2}$. In fact we have $\left[\bar{\tau}_{1,3}, \bar{\tau}_{2,2}\right]=\frac{\partial}{\partial x_{6}} \in \Delta^{T}$. Following the result presented in Section IV, if we take $\tau_{i, j}=\bar{\tau}_{i, j}$ for $j \neq 3$ and $\tau_{1,3}=\bar{\tau}_{1,3}-x_{5} \tau_{6}$, then all $\tau_{i, j}$ commute. Therefore, we can build the change of coordinates.

Step 5-7: To compute the change of coordinates, we will first calculate $\Lambda=\left(\begin{array}{ccccc}0 & 0 & 1 & 0 & 0 \\ 0 & 1 & 0 & 0 & 0 \\ 1 & 0 & x_{5} & 0 & x_{3} \\ 0 & 0 & 0 & 0 & 1 \\ 0 & 0 & -2 x_{5}-2 x_{3} & 1 & -2 x_{3}\end{array}\right)$. Then, by applying equation (31) we obtain

$$
\begin{aligned}
& d z_{1,1}=\omega_{1,1}=-x_{5} \theta_{1,1}+\theta_{1,3}-x_{3} d x_{5}=d\left(x_{1}-x_{3} x_{5}\right) \\
& d z_{1,2}=\omega_{1,2}=d x_{2}, d z_{1,3}=\omega_{1,3}=d x_{3} \\
& d z_{2,1}=\omega_{2,1}=d\left(x_{4}+2 x_{3} x_{5}\right) \\
& d z_{2,2}=\omega_{2,2}=d x_{5}
\end{aligned}
$$

Due to the fact that $\tau_{6}=\frac{\partial}{\partial x_{6}}$, hence we can simply choose $\eta=x_{6}$. Therefore we deduce the following change of coordinates:

$$
\begin{aligned}
& z_{1,1}=x_{1}-x_{3} x_{5}, z_{1,2}=x_{2}, z_{1,3}=x_{3} \\
& z_{2,1}=x_{4}+2 x_{3} x_{5}, z_{2,2}=x_{5}, \eta=x_{6}
\end{aligned}
$$

Finally, we obtain the transformed normal form as

$$
\begin{aligned}
& \dot{z}_{1,1}=y_{1}^{2}+y_{1} y_{2}, \dot{z}_{1,2}=z_{1,1}+y_{1} y_{2} \\
& z_{1,3}=z_{1,2}, \dot{z}_{2,1}=2 y_{1}^{3}, \dot{z}_{2,2}=z_{2,1}-2 y_{1} y_{2}-y_{1}^{2} \\
& \dot{\eta}=-\eta+z_{1,2} z_{2,2}, y_{1}=z_{1,3}, y_{2}=z_{2,2}
\end{aligned}
$$


Then, we can design a Luenberger-like observer of the form (10) to estimate the observable states. The corresponding simulation results are depicted in Figure 1 with the following gain:

$$
K=\left[\begin{array}{ccccc}
191.9958 & 103.9886 & 103.9886 & -0.2064 & -0.0334 \\
-9.6788 & -3.4892 & -0.3029 & 35.0112 & 12.0017
\end{array}\right]^{T}
$$

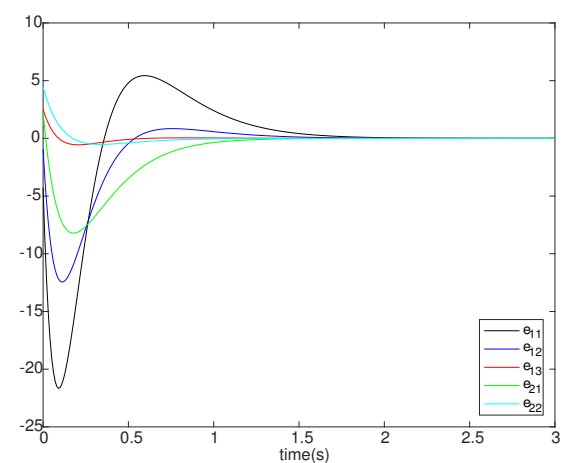

Fig. 1. Estimation errors.

\section{CONCLUSION}

This paper deduced necessary and sufficient geometric conditions to transform a nonlinear dynamical system with multiple independent outputs into a partial observer normal form. Then, a simple Luenberger-like observer can be designed for such a partial observer normal form. Examples were provided to highlight these conditions.

\section{Acknowledgment}

The authors are deeply grateful for the associated editor and the anonymous reviewers for their careful readings of our manuscript, their insightful comments and suggestions that enable us to improve considerably the writing and the scientific consistence of this work.

\section{REFERENCES}

[1] D. Bestle and M. Zeitz, "Canonical form observer design for nonlinear time varying systems," International Journal of Control, vol. 38, pp. 419-431, 1983.

[2] A.-J. Krener and A. Isidori, "Linearization by output injection and nonlinear observers," Systems \& Control Letters, vol. 3, no. 1, pp. 47-52, 1983.

[3] X. Xia and W. Gao, "Nonlinear observer with linearizable error dynamics," SIAM Journal on Control and Optimmisation, vol. 27, pp. 199-216, 1989.

[4] A.-J. Krener and W. Respondek, "Nonlinear observers with linearizable error dynamics," SIAM Journal on Control and Optimization, vol. 23, no. 2, pp. 197-216, 1985.

[5] A. Isidori, Nonlinear control systems. Springer Science \& Business Media, 2013.

[6] M. Hou and A. Pugh, "Observer with linear error dynamics for nonlinear multi-output systems," Systems \& Control Letters, vol. 37, pp. 1-9, 1999

[7] A.-F. Lynch and S.-A. Bortoff, "Nonlinear observers with approximately linear error dynamics: the multivariable case," IEEE Transactions on Automatic Control, vol. 46, no. 6, pp. 927-932, 2001.

[8] A. Phelps, "On constructing nonlinear observers," SIAM Journal on Control and Optimization, vol. 29, pp. 516-534, 1991.

[9] H. Keller, "Nonlinear observer design by transformation into a generalized observer canonical form," International Journal of Control, vol. 46, no. 6, pp. 1915-1930, 1987.

[10] A. Glumineau, C. Moog, and F. Plestan, "New algebraic-geometric conditions for the linearization by input-output injection," IEEE Transactions on Automatic Control, vol. 41, no. 4, pp. 598-603, 1996.

[11] M. Lopez, F. Plestan, and A. Glumineau, "Linearization by completely generalized input ouput injection," Kybernetika, vol. 35, pp. 793-802, 1999.

[12] W. Respondek, A. Pogromsky, and H. Nijmeijer, "Time scaling for observer design with linearizable error dynamics," Automatica, vol. 40, no. 2, pp. 277-285, 2004.
[13] M. Guay, "Observer linearization by output-dependent time-scale transformations," IEEE Transactions on Automatic Control, vol. 47, no. 10, pp. 1730-1735, 2002.

[14] G. Zheng, D. Boutat, and J.-P. Barbot, "A single output dependent observability normal form," SIAM Journal on Control and Optimization, vol. 46, no. 6, pp. 2242-2255, 2007.

[15] Y. Wang and A. Lynch, "Observer design using a generalized time-scaled block triangular observer form," Systems \& Control Letters, vol. 58, pp. 346-352, 2009.

[16] D. Boutat, A. Benali, H. Hammouri, and K. Busawon, "New algorithm for observer error linearization with a diffeomorphism on the outputs," Automatica, vol. 45, no. 10, pp. 2187-2193, 2009.

[17] P. Jouan, "Immersion of nonlinear systems into linear systems modulo output injection," SIAM Journal on Control and Optimisation, vol. 41, no. 6, pp. 1756-1778, 2003.

[18] D. Noh, N. Jo, and J. Seo, "Nonlinear observer design by dynamic observer error linearization," IEEE Transactions on Automatic Control, vol. 49, no. 10, pp. 1746-1753, 2004.

[19] J. Back, K. T. Yu, and J. H. Seo, "Dynamic observer error linearization," Automatica, vol. 42, no. 12, pp. 2195-2200, 2006.

[20] R. Tami, D. Boutat, and G. Zheng, "Extended output depending normal form," Automatica, vol. 49, no. 7, pp. 2192-2198, 2013.

[21] D. Boutat, "Extended nonlinear observer normal forms for a class of nonlinear dynamical systems," International Journal of Robust and Nonlinear Control, vol. 25, no. 3, pp. 461-474, 2015.

[22] G. Zheng, D. Boutat, and J.-P. Barbot, "Multi-output dependent observability normal form," Journal of Nonlinear Analysis Series A: Theory, Methods \& Applications, vol. 70, no. 1, pp. 404-418, 2009.

[23] _ , "Output dependent observability linear normal form," IEEE CDCECC, 2005.

[24] H. N. Jo and H. J. Seo, "Observer design for non-linear systems that are not uniformly observable," International Journal of Control, vol. 75, no. 5, pp. 369-380, 2002.

[25] K. Röbenack and A.-F. Lynch, "Observer design using a partial nonlinear observer canonical form," International Journal of Applied Mathematics and Computer Science, vol. 16, pp. 333-343, 2006.

[26] D. Luenberger, "An introduction to observers," IEEE Transactions on automatic control, vol. 16, no. 6, pp. 596-602, 1971.

[27] M. Darouach, M. Zasadzinski, and M. Hayar, "Reduced-order observer design for descriptor systems with unknown inputs," IEEE Transactions on Automatic Control, vol. 41, no. 7, pp. 1068-1072, 1996.

[28] G. Zheng and D. Boutat, "Synchronisation of chaotic systems via reduced observers," IET Control Theory \& Applications, vol. 5, no. 2, pp. 308-314, 2011.

[29] K. Zhou, J. C. Doyle, K. Glover et al., Robust and Optimal Control. Prentice Hall New Jersey, 1996, vol. 40.

[30] W. Saadi, D. Boutat, G. Zheng, and L. Sbita, "Multi-output partial nonlinear observer normal form," in 2015 54th IEEE Conference on Decision and Control (CDC), 2015, pp. 7654-7658.

[31] R. Marino and P. Tomei, Nonlinear control design: geometric, adaptive and robust. Prentice Hall International (UK) Ltd., 1996.

[32] R. Abraham, J. E. Marsden, and J. E. Marsden, Foundations of mechanics. Benjamin/Cummings Publishing Company Reading, Massachusetts, 1978, vol. 36.

[33] H. Nijmeijer and A. Van der Schaft, Nonlinear dynamical control systems. Springer, 1990.

[34] F. Thau, "Observing the state of non-linear dynamic systems," International Journal of Control, vol. 17, no. 3, pp. 471-479, 1973.

[35] L. Praly, "Asymptotic stabilization via output feedback for lower triangular systems with output dependent incremental rate," IEEE Transactions on Automatic Control, vol. 48, no. 6, pp. 1103-1108, 2003. 